\title{
Genesis
}

Manuscrits - Recherche - Invention

$44 \mid 2017$

Après le texte

\section{Duras : de quelques réécritures mineures}

\section{Gilles Philippe}

\section{(2) OpenEdition \\ Journals}

\section{Édition électronique}

URL : https://journals.openedition.org/genesis/1759

DOI : 10.4000/genesis. 1759

ISSN : 2268-1590

\section{Éditeur :}

Presses universitaires de Paris Sorbonne (PUPS), Société internationale de génétique artistique littéraire et scientifique (SIGALES)

\section{Édition imprimée}

Date de publication : 9 mai 2017

Pagination : 109-118

ISBN : 979-1023-105636

ISSN : 1167-5101

\section{Référence électronique}

Gilles Philippe, «Duras : de quelques réécritures mineures », Genesis [En ligne], 44 | 2017, mis en ligne le 16 mai 2018, consulté le 16 mars 2023. URL : http://journals.openedition.org/genesis/1759; DOI : https://doi.org/10.4000/genesis.1759 


\title{
Duras : de quelques réécritures mineures
}

\author{
Gilles Philippe
}

L 'historiographie littéraire considère à raison que la réécriture est une des caractéristiques les plus frappantes de la production de Marguerite Duras. Elle en a fait valoir les cas les plus extrêmes, ceux où un même matériau narratif, thématique ou textuel est repris dans un genre ou un sous-genre différent de celui de sa première apparition. Mais elle en a négligé les cas les plus fins, ceux où l'écrivaine est intervenue localement sur un de ses livres à l'occasion d'un retirage ou d'un passage en poche. Il est vrai que ces modifications étaient, pour la plupart, passées totalement inaperçues avant la récente parution des Euvres complètes de l'auteure1. Or, bien que le plus souvent «simplement»stylistiques, ces réécritures mineures méritent d'être mises en relation avec les cas de réécriture les plus emblématiques de l'œuvre. Dégager leur nature, leur ampleur, leur rendement et leurs enjeux permet alors de procéder à quelques ajustements interprétatifs dont l'intérêt est loin d'être seulement ponctuel.

Il serait assurément vain de chercher une quelconque unité dans les modifications opérées par Marguerite Duras à l'occasion de la republication de ses textes. S'il n'y eut ni pratique systématique, ni stratégie concertée, c'est tout d'abord pour des raisons contingentes. Les plus évidentes, ce sont sans aucun doute les circonstances personnelles, par exemple la plus ou moins grande disponibilité physique ou morale de l'auteure au moment où est enclenché le processus de retirage ou de changement de format. Des circonstances extérieures peuvent en outre expliquer que certains textes aient été revus et d'autres pas. Si l'on considère, par exemple, les trois romans que Duras a fait paraître au début des années cinquante, on voit que la réécriture est d'abord liée au fait que l'auteure ou son éditeur ont pensé qu'une adaptation filmique en préparation entraînerait un regain d'attention pour le récit. En 1958, Gallimard décida ainsi de retirer Un barrage contre le Pacifique (1950) à l'occasion de la sortie du film de René Clément, et Duras revit son texte de très près. En 1966, une nouvelle édition du Marin de Gibraltar présente de nombreux changements, attestant que la romancière en a revu le texte sans doute parce qu'Alain Resnais puis Tony Richardson avaient songé à porter à l'écran le récit de 1952. Il est dès lors fort probable que Les Petits Chevaux de Tarquinia (1953) aurait été mêmement revu si un projet de film avait été envisagé ; il n'en fut rien, de sorte que ni les retirages ni le passage en poche n'entraînèrent de modifications du texte initial.

Bien qu'il faille faire à la contingence sa part, quelques lignes fortes apparaissent lorsque l'on croise une double chronologie : celle de la première parution des œuvres et celle de leur reprise avec possible modification de leur état original. Pour bien comprendre ce qui a pu se jouer ici, il convient dès lors de garder en mémoire que l'on distingue communément trois temps dans l'ensemble du parcours littéraire de Marguerite Duras : elle a elle-même souvent proposé de considérer que l'ensemble des livres parus avant 1958 relevait d'une première manière dans laquelle elle ne se reconnaissait plus $^{2}$; une deuxième période court de 1958 à 1973, avant que le cinéma ne prenne, pendant sept ans, le pas sur l'écriture; parus après 1980, les livres de la troisième période marquent un net changement de ton, de style ou d'univers de référence.

1. Marguerite Duras, Euvres complètes (désormais $O C$ ), Paris, Gallimard, coll. «Bibliothèque de la Pléiade», t. I et II, 2011, t. III et IV, 2014, sous la direction de l'auteur de cet article, qui tire une bonne partie de ses informations des recherches scrupuleuses et des appareils critiques fournis par l'ensemble des collaborateurs. Que ceux-ci en soient vivement remerciés.

2. Voir, par exemple, Les Parleuses (1974), éd. Anne Cousseau, OC III, p. 8. 
On verra plus loin que Duras n'explique pas entièrement Duras, et que les réorientations de l'œuvre ne s'expliquent pas seulement voire pas d'abord par une réorientation de son projet esthétique personnel. Mais on note tout d'abord que les ouvrages de la deuxième période n' ont, pour ainsi dire, jamais subi aucun changement. Il est vrai que plusieurs de ces livres ont été repris dans la collection «L'Imaginaire» des éditions Gallimard, qui fournissait le plus souvent des textes non recomposés, strictement identiques à ceux de la parution originale. Tel fut le cas de L'Après-midi de Monsieur Andesmas (1962), du Vice-Consul (1966), de L'Amante anglaise (1967), ou encore d'India Song (1973), tous textes réimprimés ne varietur dans «L'Imaginaire» entre 1977 et 1991. Mais, même lorsque les modalités de la réédition autorisaient voire exigeaient la recomposition, les livres publiés entre 1958 et 1973 furent repris à l'identique, à l'exception de quelques corrections de langue (souvent de simples ajustements de ponctuation ou d'orthographe) fréquemment attribuables à un préparateur éditorial. Pour le reste, aucun vrai travail n'apparaît, ni à l'occasion d'un retirage, ni à l'occasion d'un changement de format, et cela quelle que soit la date de la republication, que celle-ci implique ou non un changement d'éditeur. Si ce n'est d'infimes retouches, rien ne distingue ainsi le texte original et le texte final des trois récits parus en 1960, Moderato cantabile (en «10/18 » dès 1962, en «Double» chez Minuit en 1980), Hiroshima mon amour et Dix heures et demie du soir en été (en «Folio» dès 1971 et 1985). De la même manière, bien que seize ans séparent le passage en poche du flamboyant Ravissement de Lol V. Stein (1964, «Folio » 1976) et celui du très austère L'Amour (1971, «Folio» 1992), il semble que Duras n'ait jamais songé à revoir ces deux livres, pas plus qu'elle ne souhaita modifier le mystérieux Détruire dit-elle de 1969, lorsqu'en 1972 celui-ci fut repris dans la collection «10/18».

En de telles circonstances, la raison la plus simple est souvent la meilleure : Duras s'estimait sans doute pleinement satisfaite de l'ensemble des textes de cette deuxième période de son œuvre, que l'on retient de fait, et peut-être à raison, comme présentant le sommet de sa production littéraire. Mais ce constat oblige à s'interroger sur la moindre stabilité du parcours éditorial des textes de la première et surtout de la troisième période de l'œuvre.

\section{Creuser l'écart : le retravail des récits de la première période}

Si les deux premiers romans de Marguerite Duras ne furent jamais retouchés, c'est d'abord pour des raisons presque inverses : elle n'eut ni la force ni l'envie de se replonger dans Les Impudents (1943, en «Folio » dès 1992); elle garda toujours une tendresse et une fierté pour La Vie tranquille (1944, en «Folio» dès 1981). Mais les altérations apparues lors des réimpressions intervenues, dans les circonstances rappelées plus haut, entre le premier et le dernier état d'Un barrage contre le Pacifique et du Marin de Gibraltar autorisent à émettre une hypothèse intéressante. Loin d'aligner la norme rédactionnelle de ces deux romans sur la «nouvelle manière» de Duras, ces altérations les en éloignent et contribuent à creuser l'écart stylistique entre la première et la deuxième période de l'œuvre de l'écrivaine. Ainsi celle-ci supprima-t-elle par exemple quelques répétitions malheureuses, alors qu'elle devait revendiquer plus tard la répétition comme l'une de ses plus notables et de ses plus anciennes marques de fabrique stylistiques.

L'étude systématique des modifications intervenues entre l'originale de 1950 et l'état de 1958 d'Un barrage contre le Pacifique a permis à Julien Piat de faire apparaître que Marguerite Duras a retravaillé son texte dans trois directions parfaitement congruentes : elle a d'une part - soit concession, soit correction - estompé certaines maladresses («dans un de ses doigts» devient par exemple «à l'un de ses doigts»); elle a d'autre part élevé le niveau lexical de la narration («se décider» est par exemple remplacé par «se résoudre», «ayant l'air de» par «paraissant», «avaient» par «possédaient», etc.); elle a enfin également élevé le standard grammatical de sa prose, en substituant ponctuellement le subjonctif plus-que-parfait au conditionnel composé $^{3}$. Ce travail est cependant resté incomplet, tout comme la nouvelle campagne d'ajustements normatifs (peutêtre due à une main tierce, mais sans doute autorisée par l'écrivaine) intervenue à l'occasion du passage en «Folio » de 1977. Ce double manque de rigueur fait que nous lisons aujourd'hui un texte qui présente localement des sautes

3. Voir Julien Piat, Note sur le texte d'Un barrage contre le Pacifique (1950), OC I, p. 1475. 
et donc une couleur stylistiques que son auteure n'avait probablement pas souhaitées.

Marguerite Duras a procédé à des modifications de même nature lorsqu'elle a repris Le Marin de Gibraltar en vue de sa reparution dans la collection «Soleil» en 1966. Certes, cette fois, la romancière ajouta ou supprima quelques segments, alors qu'elle s'en était tenue à de simples substitutions pour le Barrage. Elle n'eut point, en outre, à élever le niveau lexical ou grammatical du récit, puisque sa prose avait ici bien moins cédé à l'attraction des conventions «oralisantes » romanesques qui avaient, dans le livre précédent, largement débordé des répliques vers la langue de la narration. Bien que les modifications soient largement cantonnées dans le tout début du roman (comme si la tâche de révision avait été rapidement abandonnée), il reste évident que Duras voulut à nouveau assagir et unifier le style du livre, soit en corrigeant ce qui pouvait sembler malvenu («Le vent était d'une frâ̂cheur extraordinairement bonne» devint ainsi «Le vent était d'une fraîcheur délicieuse $\left.{ }^{4} \gg\right)$, soit, et presque à l'inverse, en renonçant à de possibles préciosités : «il me fallait un fleuve, de l'eau d'ombre» laisse ainsi place à «il me fallait un fleuve, de l'eau sous l'ombre des arbres ${ }^{5} »$. Cette «eau d'ombre», pour « cette eau sombre», rappelait sans doute trop un certain «style 1900» qui avait baigné les premières lectures de Duras : l'historiographie stylistique retient d'ailleurs que nul n'avait plus fréquemment délaissé l'épithète au profit du génitif de caractérisation que Pierre Loti, cet auteur aux «forêts d'ombre» dont les livres avaient enchanté l'enfance de la jeune Indochinoise, comme elle se plut toujours à le rappeler. L'entrée du Marin dans la collection «Folio», en 1977 également, donna lieu, comme celle du Barrage, à une nouvelle campagne d'ajustements normatifs.

La congruence des changements intervenus au cours du trajet éditorial d'Un barrage contre le Pacifique et du Marin de Gibraltar n'apparaît cependant pleinement que si l'on prend en considération un autre texte qui ne leur est postérieur que de quelques années. Bien que Duras ait validé la date de 1958 comme borne a quo de sa nouvelle période (sans doute parce que, pour la première fois, elle était passée chez Minuit), il serait plus exact de considérer qu'elle avait renoncé à sa première esthétique dès 1955 , année où elle avait donné un roman dialogué, Le Square, dont l'esprit et la manière regardaient déjà nettement vers certains livres à venir. Cette fois, les modifications sont bien plus tardives, puisqu'elles n'apparaissent qu'avec le passage en poche de 1990. Si certaines corrections de langue doivent, par prudence, être attribuées à un relecteur, les altérations stylistiques ne peuvent être rapportées qu'à l'auteure. Celles-ci restent rares, certes, mais elles sont fort instructives, comme on peut en juger sur les premières lignes du texte. En 1955, on avait pu lire ceci : «L'enfant arriva tranquillement du fond du square et se planta devant la jeune fille. / "J'ai faim”, déclara l'enfant. » Depuis 1990, le texte s'ouvre ainsi : «Tranquillement, l'enfant arriva du fond du square et se planta devant la jeune fille. / "J'ai faim", dit $l^{\prime}$ 'enfant ${ }^{6}$. Or, le déplacement de l'adverbe semble étrange : celui-ci était mieux venu après le verbe; détaché en tête de proposition, il donne en outre le sentiment d'arrimer la phrase qui suit à celle qui précède, ce qui crée un effet d'attaque in mediam orationem. Il y a ici quelque chose de gauche voire de gauchi, de mal écrit ou du moins de désécrit, que confirme encore la substitution de «dit» à «déclara», c'est-à-dire du verbe de parole le plus banal à l'un de ses hyponymes plus recherchés (à l'inverse, par exemple, du remplacement d' «avaient» par «possédaient» noté plus haut dans Un barrage).

Or, ce même appauvrissement du lexique s'observe ailleurs et notamment dans les répliques. Ainsi avait-on lu en 1955 : «Et je ne vois pas non plus de quel côté de ma vie cette chance pourrait me venir, d'où elle pourrait souffler. Je ne veux pas dire qu'elle ne pourrait pas souffler un jour, n'est-ce pas, on ne peut jamais savoir, ni que, si elle soufflait, je ne l'accueillerais pas volontiers [...]»; le texte de 1990 devait substituer arriver aux trois occurrences du verbe souffler ${ }^{7}$. Peut-être Duras jugea-t-elle la métaphore médiocre, mais surtout elle préférait désormais les termes neutres aux termes sémantiquement plus denses, et sans doute apprécia-t-elle aussi l'effet de maladresse ainsi créé : une répétition est jugée oratoire et non fautive dès

4. Le Marin de Gibraltar, OC I, éd. Bernard Alazet, p. 540 (pour le texte original : Paris, Gallimard, 1952, p. 25).

5. Ibid., p. 544 (édition originale, p. 29). Ces modifications, repérées par B. Alazet, n'ont pu être reprises dans la Pléiade.

6. Le Square (1955), OC I, p. 1137 ; pour la variante, voir la notice de Brigitte Ferrato-Combe, p. 1552, n. 2. Ces phrases se retrouvent presque à l'identique à l'ouverture de chacune des trois sections; Duras a donc procédé trois fois aux mêmes modifications.

7. Ibid., p. 1140 et note p. 1552. 
lors qu'elle est exhibée, mais maladroite et condamnable si elle n'est rattachable à aucun projet figural. En attestent les nombreux pastiches des années quatre-vingt : le ressassement des mêmes mots banals et abstraits (dire, aller, voir...) était désormais tenu pour l'une des principales signatures stylistiques de l'écrivaine.

Ces quelques modifications n'expliquent bien sûr pas à elles seules que nous soyons aujourd'hui tentés de placer en 1955 plutôt qu'en 1958 la principale rupture intervenue dans l'esthétique romanesque de Marguerite Duras. Elles renforcent en tout cas le sentiment de parenté que nous pouvons avoir à la lecture du Square et de textes bien plus tardifs. Car il apparaît clairement que, lorsqu'elle prépara en 1989 le passage en poche de ce roman, l'auteure voulut accentuer le côté «durassien» de sa prose. Ouvert en 1969 ( Je ne peux plus du tout lire un roman. À cause des phrases $\left.{ }^{8} \gg\right)$, largement instruit à l'occasion de la parution de L'Amant en 1984, le procès des conventions de la phrase romanesque (la distribution soignée des constituants, la richesse du lexique, l'étoffement des groupes, etc.) faisait depuis longtemps partie de la «posture Duras» et faisait également allégeance aux nouvelles valeurs stylistiques du moment : «La plupart des romans refusés par les éditeurs sont des romans trop écrits. Queneau me disait : il faut se méfier des romans. Un roman d'une écriture parfaite, écrite jusqu'à son plein, c'est à fuir ${ }^{9}$. »

Avec une partie des écrivains de son temps, Duras avait commencé à négocier son virage vers le «moins écrit» dès 1957. Cette année-là, elle avait notamment lu Le Bleu $d u$ ciel dont elle rédigea un éloge qui devait paraître en janvier 1958 : «On peut donc dire de Georges Bataille qu'il n'écrit pas du tout puisqu'il écrit contre le langage. Il invente comment on peut ne pas écrire tout en écrivant. Il nous désapprend la littérature. L'absence de style du Bleu du ciel est un ravissement. [...] Comment peut-on ne pas écrire à ce point 10 ?» Le programme esthétique ici affiché semble l'exact inverse de celui qui gouvernait, sans doute exactement au même moment, les retouches stylistiques du Barrage, tout l'inverse aussi de celui qui gouvernerait, quelques années plus tard, la reprise du Marin de Gibraltar. Même si la stratégie fut sans doute moins calculée que pour la reprise du Square en 1990, tout porte à croire que la réécriture de ces deux romans a aussi visé à les confiner dans une période désormais révolue, celle des livres «si simples et si charmants », pour reprendre des mots que Duras ne pardonna jamais à Roland Barthes ${ }^{11}$, livres qu'elle-même avait pourtant, inconsciemment ou volontairement, cherché à rendre toujours plus tels à l'occasion de leur nouvelle parution.

On l'a dit plus haut, Duras n'explique pas entièrement Duras. Le changement intervenu dans sa prose au cours des années cinquante est exactement contemporain d'une bascule générale de la norme stylistique, le «bien écrit»se voyant soudain dévalorisé et associé à un certain style NRF que récuse vigoureusement la «littérature restreinte», celle qui prétend à une valeur esthétique élevée. Ainsi pourrait-on, exemple parmi d'autres, mettre en parallèle le trajet stylistique effectué par Julien Gracq entre Le Rivage des Syrtes (1951) et Un balcon en forêt (1958) et celui qu'effectue Duras au cours des mêmes années ${ }^{12}$. Ainsi est-il frappant qu'apparaissent dans Moderato cantabile en 1958 des «lourdeurs» comme «Anne Desbaresdes aussi reconsidéra cet enfant de ses pieds jusqu'à sa tête ${ }^{13}$ » (le français évite le possessif avec les parties du corps, et l'on attend «des pieds à la tête» ou, mieux encore, « de la tête aux pieds»), alors même que Duras pourchasse au même moment ce type de maladresse en reprenant le texte d'Un barrage contre le Pacifique.

\section{Réduire l'écart : la genèse continuée des récits de la dernière période}

Par ses modalités et ses enjeux, le trajet éditorial des récits postérieurs à 1980 diffère assez radicalement de ce que nous avons observé pour les romans parus avant 1958. Sans doute trouve-t-on encore ici tout un ensemble de textes dont

8. «La destruction de la parole», Les Cahiers du cinéma, no 217, 1969, p. 45. Sur l'évolution de l'écriture de Duras, voir Sandrine Vaudrey-Luigi, La Langue romanesque de Marguerite Duras, Paris, Classiques Garnier, 2013

9. «Flaubert c'est...» (1982), Le Monde extérieur (1993), éd. Joëlle Pagès-Pindon, $O C$ IV, p. 934.

10. «À propos de Georges Bataille» (1958), Outside (1981), éd. Robert Harvey, OC III, p. 886-887.

11. Yann Andréa Steiner (1992), éd. Chloé Chouen-Ollier, OC IV, p. 781. 12. Sur cette mutation de la norme stylistique au milieu des années cinquante, voir G. Philippe, Le Rêve du style parfait, Paris, Presses universitaires de France, 2013, passim.

13. Moderato cantabile (1958), éd. B. Ferrato-Combe, OC II, p. 1206. 


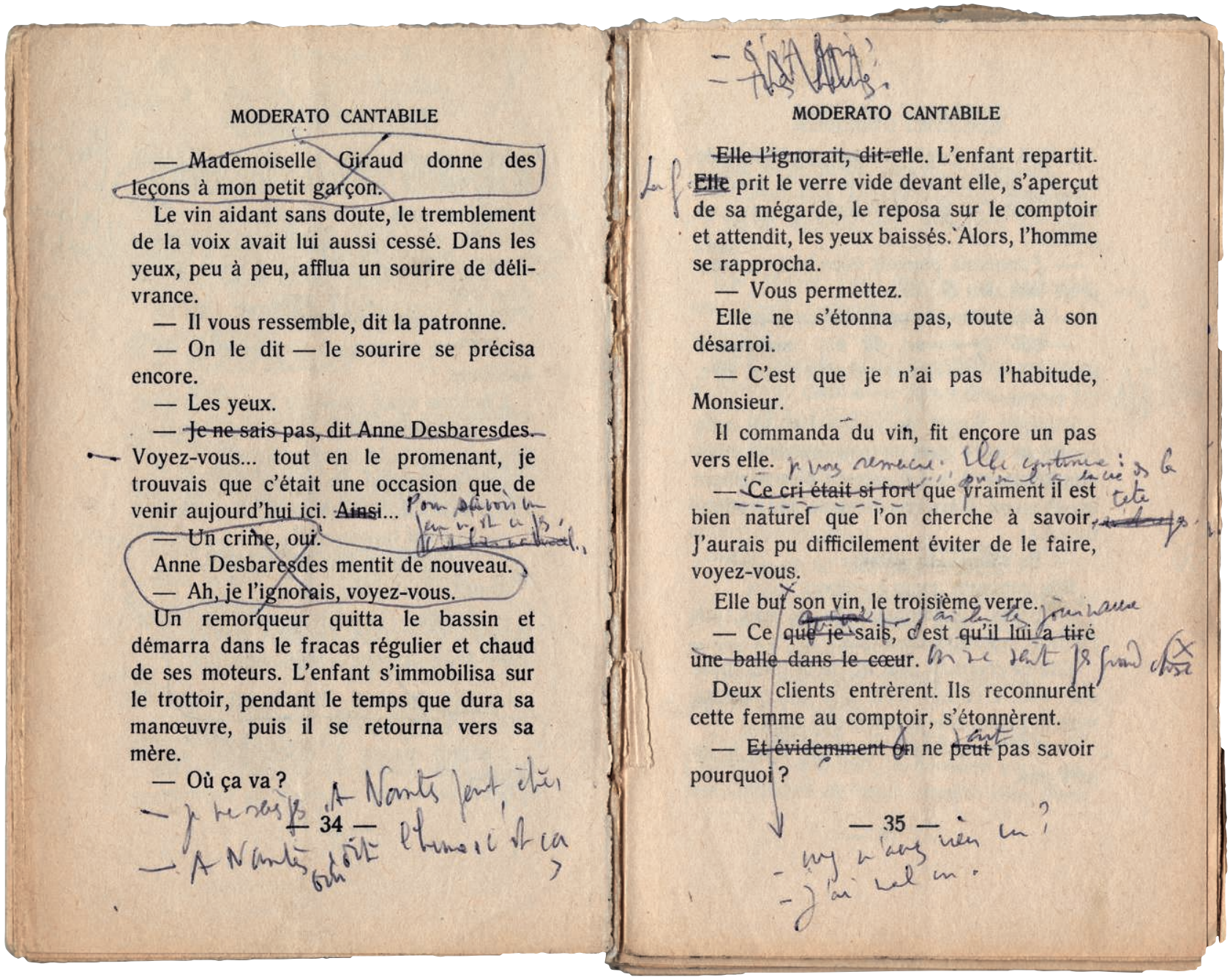

Fig. 1 : Exemplaire de l'édition originale de Moderato cantabile (Minuit, 1958) avec des corrections manuscrites qui ne furent pas prises en compte pour les tirages postérieurs (Fonds Marguerite Duras/IMEC, DRS 23.405)

l'édition originale s'est avérée définitive : tel est ainsi le cas de L'Homme assis dans le couloir (198114), de L'Homme atlantique et de La Maladie de la mort (1982), ou encore des Yeux bleus cheveux noirs (1986) et de La Pluie d'été (1990). Sans doute faut-il aussi faire à nouveau la part de la contingence : peut-être Marguerite Duras eût-elle été tentée de revoir le texte d'Emily L. (1987), mais l'ambitieux premier tirage était loin d'être épuisé au moment de sa mort. Sans doute ne faut-il pas en outre chercher à comprendre les enjeux des modifications opérées dans les textes romanesques sur le modèle de ce que nous offrent les textes dramatiques de la même époque. On en connaît le cas le plus célèbre : Savannah Bay, dont une première édition parut en septembre 1982, qui laissa place, un an plus tard, à une «nouvelle édition augmentée», qui doublait le texte original d'une seconde version sensiblement différente, plus resserrée et plus théâtrale, issue du travail scénique intervenu en vue de la création de la pièce en septembre 198315 .

Mais trois textes narratifs de la dernière période de Marguerite Duras témoignent d'une genèse prolongée au-delà de la première édition, à l'occasion de retirages espacés de plusieurs semaines ou de plusieurs mois, et non

14. Une première version en avait paru dans L'Arc en 1962, mais l'on n'évoquera pas ici le cas bien différent des quelques textes narratifs partiellement ou entièrement publiés en périodique avant reprise en volume.

15. Voir A. Cousseau, Notice de Savannah Bay (1982-1983), OC III, p. 1803. Pour une comparaison avec les deux versions publiées de L'Éden Cinéma (1977, devenu Éden Cinéma en 1988), voir la Notice de J. Piat (OC III, p. 1648-1649). Les textes théâtraux posent des problèmes très spécifiques qui ne seront pas abordés ici (voir par exemple La Musica Deuxième, réécriture en 1985 de la pièce de 1965). 


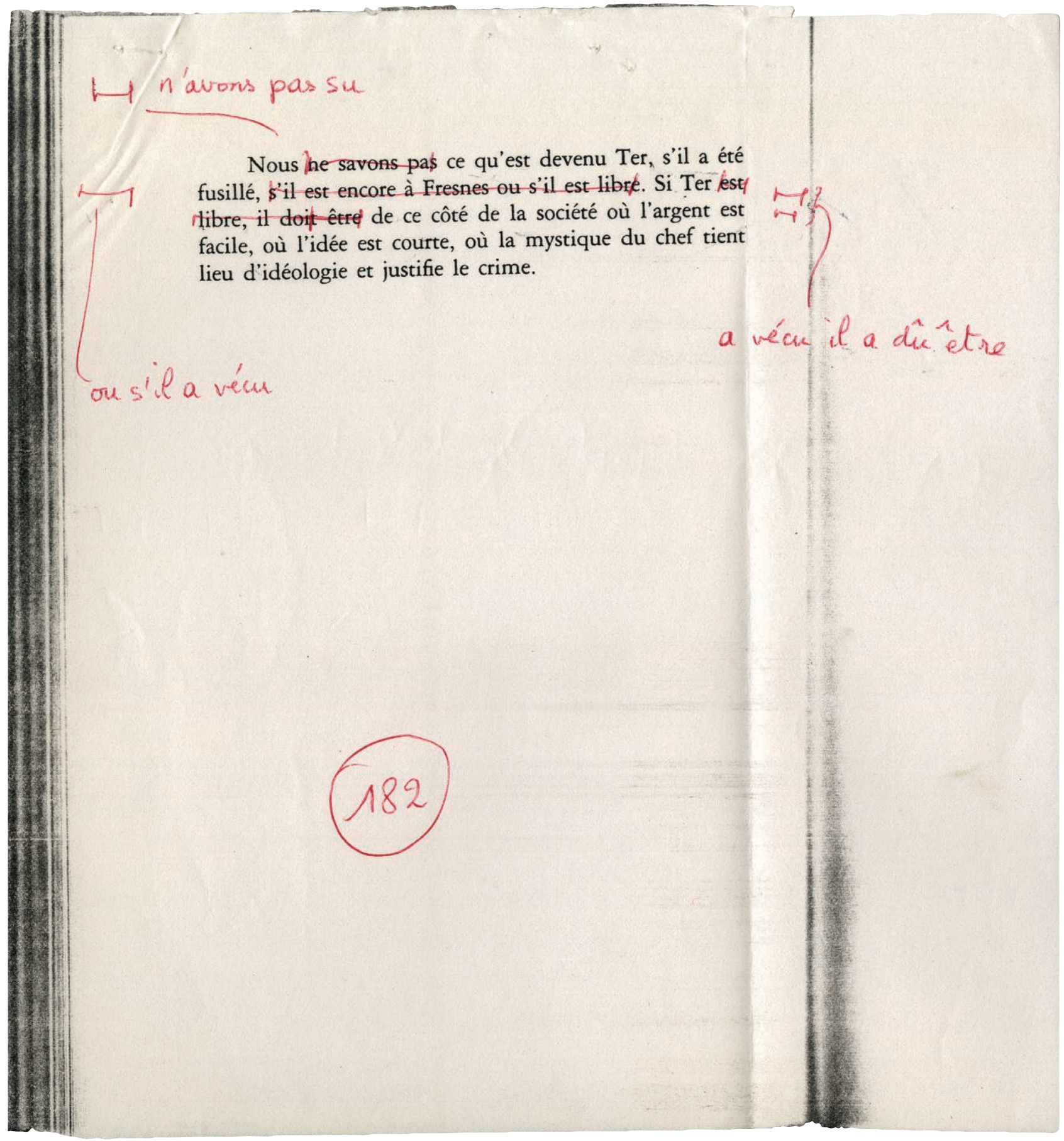

Fig. 2 : Exemplaire de l'édition originale de La Douleur (P.O.L, 1985) avec quelques corrections allographes (dictées par l'auteure ou reportées par un tiers) qui ont été intégrées aux tirages postérieurs (Fonds Marguerite Duras/IMEC, 76/DRS/15/17) 
D URAS : D E Q UEL Q UES RÉÉCRIT URES M INEURES

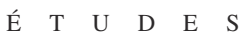

(1) $P 110$

Lo senue fille a dit sn'elle ne savait pas er queaps, bs de pours Jufs 'es avaunt chaaté

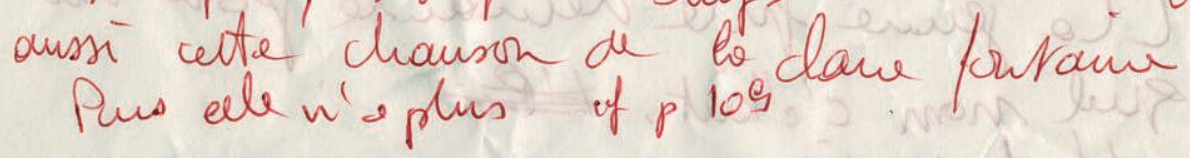

(2) $\rho \mu x$

Pans l'enfant avart demandí en wer porn ts suis. Lo geme plle n'arat pos ie poudu.

113 L'Enfaur ne plemart pas, If essayait de se souvenir et il a souvenait, Il rocourair que-(lesaif)

He ye fuoi i fer reppetait incre

Elle len avait deunandé lo caclen os feve. It m savart plus. Vert, I noypur, se mue le disait

Un cuir fur ne o'ecur pas, qui X parle senleurent, ents ls Jirls, depmis dura mille aus (Lepurts ceur millé ans) or we sair posa

Fig. 3 : Feuillet manuscrit présentant des ajouts qui interviendront entre la première et la deuxième édition de Yann Andrea Steiner (P.O.L, 1992) (Fonds Marguerite Duras/IMEC, 76DRS/36/5)

(115) 
de plusieurs années voire de plusieurs décennies comme pour les romans de la première période. Le cas le moins connu concerne pourtant le livre le plus célèbre de l'auteure. L'Amant paraît en juillet 1984; le succès est immédiat; une réimpression est rapidement programmée pour le début de septembre, et Duras peut modifier ponctuellement son texte. Elle opère d'abord quelques substitutions, dont le cas le plus intéressant est proche de l'ouverture. On avait lu en juillet : «Tout a commencé de cette façon pour moi, par ce visage voyant, exténué, ces yeux cernés en avance sur le temps, les faits $16_{» ;}$; la version de septembre devait remplacer ces deux derniers mots par «l'experiment». Sans doute Duras faisait-elle erreur sur le sens du terme anglais, dont elle crut qu'il correspondait au terme français l'expérience (vécue), dont il aurait gardé la dénotation existentielle sans en avoir les connotations morales. «Les faits» saisissait la vie de l'extérieur et l'émiettait, «l'experiment» en faisait une substance vivante se déployant dans la durée, ce qui convenait mieux au projet du livre. Et puis Duras se plut toujours à donner un petit air anglais à sa prose. Ailleurs, elle amplifia légèrement son texte. Le dernier paragraphe du roman rapporte ainsi que, bien après les événements qui font le cœur du récit, l'ancien amant avait appelé la narratrice; Duras étoffa les propos de l'homme par l'ajout de deux brèves phrases («Et aussi pour le petit frère, qu'il avait été triste pour elle. Et puis il n'avait plus su quoi lui dire $\left.{ }^{17} »\right)$, qui réactivent la thématique incestueuse mais permettent surtout de retarder et donc de rendre plus sensible encore la déclaration d'amour sur laquelle l'ouvrage s'achève.

En 1990, Marguerite Duras réécrivit complètement $L$ 'Amant pour en donner une version à couleur cinématographique qui devait paraître en 1991 sous le titre L'Amant de la Chine du Nord. Le livre passa en «Folio» dès 1993, ce qui permit à l'auteure d'en revoir le texte, qu'elle modifia à une quinzaine d'endroits. Le principal changement tient dans la suppression d'un passage de trois cents mots environ, cas unique dans le trajet éditorial des récits de Duras 18 . Elle voulut, semble-t-il, principalement abréger un dialogue qui lui apparut un peu long et redondant, et l'on peut en effet dire qu'en règle générale, les altérations introduites visent à alléger le texte et à en simplifier la prose. Ainsi la romancière supprima-t-elle les deux derniers mots de la phrase suivante : «L'enfant et le petit frère pleurent l'un contre l'autre, scellés dans un désespoir de sang 19 .» Relisant ces deux passages : «le cri tragique de son bonheur» et «comme la folie, la mort, comme une folie illisible», elle fut peut-être gênée par leur grandiloquence, puisqu'elle les atténua en «le cri de délivrance de son fils» et «comme la folie, la mort, la passion $20_{\text {». }}$.

Dans tous les cas que l'on a évoqués jusqu'à présent, les modifications postérieures à la première édition ont été faites de façon tacite, sans que le nouveau volume ne signale que des changements sont intervenus. Cela rend d'autant plus spectaculaire l'histoire éditoriale du tout dernier texte narratif de Marguerite Duras, Yann Andréa Steiner. P.O.L avait fait imprimer le livre le 9 juin 1992, mais la réimpression du 24 juin porte déjà la trace d'un important travail de révision, bientôt suivi d'une autre salve de modifications, puisque l'impression du 7 juillet (plus tard reprise en «Folio») est dite «définitive». Les changements opérés sont ici très nombreux et de tous ordres, même s'ils ne vont pas au-delà de l'ajout de quelques lignes et si les suppressions sont rares et ne portent guère que sur des mots isolés.

Le travail qui s'observe entre les trois versions du texte ne fait guère que prolonger celui que Duras avait effectué en corrigeant les épreuves ${ }^{21}$. La comparaison entre la première et la dernière version du livre opérée par Rudolf Mahrer à l'aide du logiciel MEDITE22 fait cependant clairement apparaître que c'est surtout le dernier tiers du récit qui a été modifié. On note parfois l'alignement du texte sur la norme stylistique désormais usuelle de l'auteur: le verbe écrire peut, par exemple, être remplacé par le verbe dire, plus vague et souvent déjà surreprésenté en contexte étroit. Mais surtout le texte final est sensiblement plus douloureux que le texte initial, et la thématique juive y est bien plus soulignée. L'édition du 24 juin fait par exemple apparaître les phrases suivantes, absentes de l'édition originale : «Et qu'elle a crié tout bas un mot que l'enfant a reconnu et qu'il a crié à son tour. Un mot qui ne s'écrit pas, qui se parle seulement entre

16. L'Amant (1984), éd. Sylvie Loignon, OC III, p. 1458 et note p. 1869. 17. Ibid., p. 1524 et note p. 1874

18. L'Amant de la Chine du Nord (1991), éd. S. Loignon, OC IV, p. 761-762 et notule p. 1449 .

19. Ibid., p. 718 et note p. 1446 .

20. Ibid., p. 726 et 722 et notes p. 1446.

21. Voir Ch. Chouen-Ollier, Notice de Yann Andréa Steiner, OC IV, p. 1451.

22. Voir ci-dessus, p. 34. 
les Juifs, depuis dix mille ans, depuis cent mille ans, on ne sait pas ${ }^{23}$. 》 Dès cette deuxième édition, le nom même de certains personnages prend une consonance judaïque. Duras devait s'en expliquer au début de juillet à une journaliste qui lui en faisait la remarque : «J'ai envie que tout le monde soit juif 24 .»

Les modifications de ce type sont bien trop nombreuses pour être ici reprises 25 , et l'explicit du récit peut suffire à nous en fournir une attestation éclairante. Le 9 juin, le texte se terminait ainsi : «L'écriture s'était fermée avec son nom. Son nom à lui seul c'était toute l'écriture de Théodora Kats. Tout était dit avec. Ce nom.» Le 24 juin, Duras ajouta encore ceci : «Et le blanc des robes. Et le blanc des pages. / C'était peut-être quelque chose d'inconnu, cette écriture-là. » On comprend qu'elle ne s'en satisfît pas, puisque cette nouvelle version semblait reproblématiser le récit en une réflexion sur l'écriture. Elle réécrivit alors la dernière phrase, si bien que le dernier paragraphe put se lire ainsi le 7 juillet : «C'était peut-être quelque chose d'encore inconnu, Théodora Kats, un nouveau silence de l'écriture, celui des femmes et des Juifs. » L'écriture confine cette fois au silence (la dernière Duras aima, presque à l'excès, jouer avec ce genre d'image dont les pasticheurs purent se délecter), mais surtout le nom de Théodora Kats est répété et, par un geste militant voire politique, ses deux traits les plus évidents sont dégagés et exhibés : la féminité et la judéité.

On voit que c'est souvent la fin de ses récits que Duras a eu soin de modifier, avec une certaine tendance à «fermer» le texte comme pour en préciser l'interprétation qu'il convenait d'en donner. En 1990, la nouvelle version du Square avait par exemple supprimé deux phrases de l'édition originale qui laissaient plus incertain l'avenir de la relation de la petite bonne et du voyageur de commerce ${ }^{26}$. On est désormais loin de l'invitation à la prolifération interprétative que Duras avait si souvent appelée de ses vœux pour les textes de sa deuxième période ${ }^{27}$. Et l'on ne saurait que superficiellement rapprocher les modifications intervenues entre les trois éditions de Yann Andréa Steiner des changements, eux aussi très nombreux et très systématiques, opérés entre la publication de la nouvelle «Le Boa» dans Les Temps modernes en octobre 1947 et sa reprise en 1954 dans le recueil Des journées entières dans les arbres. Le texte de la pré-originale était scandé par le mot cancer dont les vingt-six occurrences ont été soigneusement supprimées pour la parution en volume ou bien remplacées par des termes comme malheur, regret ou mort 28 ; la nouvelle en était devenue très abstraite, jouant d'un allégorisme qui ouvrait la porte à toutes sortes d'interprétations. Rien de tel en 1992, et il est d'ailleurs probable que c'est parce que la presse n'avait pas ou pas assez été sensible à la dimension juive de Yann Andréa Steiner, dont elle n'avait souvent retenu que la virtuosité de l'écriture ${ }^{29}$, que Duras voulut si fortement souligner cette dimension lors de la deuxième édition. Le 25 juin encore, elle avait été fort agacée par un article de Libération qui avait glissé sur ce thème à la lecture de la première version du texte ${ }^{30}$, ce qui explique peut-être qu'elle voulut en procurer une troisième version, qui devait s'achever non plus sur «cette écriture-là», mais sur le mot «Juifs».

À l'exception sans doute du cas si spectaculaire de Yann Andréa Steiner, les modifications opérées par Marguerite Duras à l'occasion de la republication de certains de ses textes peuvent sembler anodines. Elles apparaissent assurément bien maigres au regard des grandes réécritures qui caractérisent l'œuvre dans son entier et qui impliquent souvent un changement de genre voire de médium. Mais on notera que ces «réécritures mineures» se font bien plus fréquentes pour la dernière partie de l'œuvre, celle précisément où les «réécritures majeures» sont les plus nombreuses et dont il convient dès lors de les rapprocher ${ }^{31}$. En regard du travail effectué lors de la genèse prééditoriale de l'œuvre, les ajustements postérieurs à l'originale semblent également dérisoires. Pourtant ces

23. Yann Andréa Steiner, OC IV, p. 885 et note p. 1461. Les ajouts sont moindres pour l'édition du 7 juillet, mais ils vont dans le même sens. 24. «Extrait d'un entretien avec Patricia Martin», $O C$ IV, p. 835.

25. On en trouvera une sélection dans l'édition procurée par Ch. ChouenOllier pour la Pléiade.

26. Voir B. Ferrato-Combe, Notice du Square, OC I, p. 1544

27. Voir, par exemple, G. Philippe, Notice de Détruire dit-elle (1969), OC II, p. 1794-1795, et l'entretien cité p. 1167.

28. Voir R. Harvey, Notice de Des journées entières dans les arbres (1954), OC I, p. 1529.

29. Voir Ch. Chouen-Ollier, Notice de Yann Andréa Steiner, OC IV, p. 1450-1451.

30. Ibid., p. 1454.

31. Voir G. Philippe, «Avertissement», OC IV, p. XIX. 
altérations tardives méritent également notre attention, parce qu'elles sont précisément le lieu où se vérifie le mieux l'orientation interprétative que l'auteur souhaite donner à son livre et qu'elles attestent la prise en considération de la première réception de celui-ci. Ainsi le cas de Duras est-il particulièrement révélateur d'une sorte de volonté de gérer la lecture de son œuvre dans son entier : il est en effet tout à fait étonnant que les textes de sa deuxième période ne furent jamais revus, tandis que la romancière semble avoir voulu creuser l'écart entre les récits de sa première période et ceux qu'elle écrivit après 1955, et qu'elle semble s'être surtout inquiétée de la stabilisation de la dernière partie de son travail, en une sorte de geste de reprise où se joue une certaine image d'elle-même et qui a quelque chose de testamentaire.

GILles PhILIPPE est professeur de linguistique française à l'Université de Lausanne. Ses travaux portent principalement sur les pratiques stylistiques et les imaginaires langagiers aux XIX et XXe siècles. Il est notamment l'auteur de Le Rêve du style parfait (Paris, Presses universitaires de France, 2013) et de French Style. L'accent français de la prose anglaise (Bruxelles, Les Impressions nouvelles, 2016). Il collabore régulièrement à la «Bibliothèque de la Pléiade» des éditions Gallimard.

gilles.philippe@unil.ch 


\section{Duras : de quelques réécritures mineures}

L'historiographie littéraire considère à raison que la réécriture est une des caractéristiques les plus frappantes de la production de Marguerite Duras. Elle en a fait valoir les cas les plus extrêmes, ceux où un même matériau narratif, thématique ou textuel est repris dans un genre ou un sous-genre différent de celui de sa première apparition. Mais elle en a négligé les cas les plus fins, ceux où l'écrivaine est intervenue localement sur un de ses livres à l'occasion d'un retirage ou d'un passage en poche. Il est vrai que ces modifications étaient, pour la plupart, passées totalement inaperçues avant la récente parution des Euvres complètes de l'auteure. Or, bien que le plus souvent «simplement» stylistiques, ces réécritures mineures méritent d'être mises en relation avec les cas de réécriture les plus emblématiques de l'œuvre. Dégager leur nature, leur ampleur, leur rendement et leurs enjeux permet alors de procéder à quelques ajustements interprétatifs dont l'intérêt est loin d'être seulement ponctuel.

"Rewriting" is commonly and rightly regarded as one of the most distinctive features of Marguerite Duras's literary productions. But the emphasis is usually laid upon the sole spectacular examples, when some narrative, thematic or textual elements are recycled into a different work, usually belonging to another genre, subgenre or even to another medium. Less noticeable cases have been consequently overlooked, notably the minute changes made by the writer when revising one of her novels about to be reprinted or published in another format. As a matter of fact, most of these changes remained completely unnoticed until the very recent critical edition of Duras's Complete Works. Yet, even though most of the time "purely" stylistic, such minor amendments ought to be thought together with the best-known cases of rewriting retained as emblematic of Duras's career. Assessing the nature, the extent, the impact of the modifications performed by the author when one of her books was to be republished may indeed open some interesting interpretive perspectives.

Zu Recht sieht man im Überarbeiten einen der charakteristischen Züge von Marguerite Duras ' literarischer Produktionsweise. Doch dabei bezieht man sich meist auf einzelne herausragende Beispiele, wo einzelne erzählerische Elemente, thematische oder Text-Einheiten eine Wiederverwertung in einem anderen Werk erfahren, das einer anderen Gattung, Sub-Gattung angehört. Man übersah indes weniger ins Auge springende Fälle, insbesondere die kleinen Veränderungen, die die Schriftstellerin vornahm, wenn sie eines ihrer Bücher für eine Neuauflage oder für die Übernahme in eine Taschenbuchausgabe verbesserte. Tatsächlich blieben diese Textrevisionen großteils unbemerkt, bis kürzlich die kritische Ausgabe von Duras' Gesammelten Werken erschien. Und doch sollten solche kleinen Revisionen - obzwar vorwiegend „rein“ stilistisch - mit den bekanntesten Fällen von Duras“ Überarbeitungen zusammengedacht werden. Es können sich interessante Interpretationsperspektiven daraus ergeben, dass man die Natur, den Umfang, die Wirkung und den Gegenstand der Modifikationen untersucht, die die Autorin bei der Neuveröffentlichung ihrer Bücher vornahm.

La historiografía literaria considera, con razón, que la reescritura es una de las características más sorprendentes de la producción de Marguerite Duras. La escritora misma ha puesto de relieve los casos más extremos -aquellos en los que un mismo material narrativo, temático o textual ha sido retomado en un género o subgénero diferente del de su primera aparición-, pero ha subestimado los casos más delicados: aquellos en que la escritora ha intervenido localmente en uno de sus libros, con ocasión de una reimpresión o de una reedición en libro de bolsillo. Es verdad que estas modificaciones pasaron totalmente desapercibidas hasta la reciente publicación de las Obras completas de la autora. Sin embargo, si bien son a menudo "simplemente" estilísticas, estas reescrituras menores merecen ser vinculadas con los casos de reescritura más emblemáticos de la obra. Desglosar su naturaleza, amplitud, rendimiento, significaciones permite proceder a algunos ajustes interpretativos cuyo interés está lejos de ser solamente ocasional.

A historiografia literária tem razão em considerar que a reescrita é uma das características mais marcantes da produção de Marguerite Duras. Foram destacados os casos mais extremos, aqueles em que o mesmo material narrativo, temático ou textual é retomado em um género ou sub-género diferente do da primeira aparição. Mas passaram em claro casos menos evidentes, em que a escritora interveio localizadamente em um dos seus livros, aquando de uma reimpressão ou alteração de formato. Tendo passado totalmente despercebidas antes da recente publicação das Obras completas da autora, estes pequenos retoques merecem ser postos em confronto com os casos mais emblemáticos. Perceber a sua natureza, o seu alcance, o seu desempenho e os seus problemas permitirá fazer alguns ajustes interpretativos, não destituídos de interesse.

La storiografia letteraria considera a ragione la riscrittura come una delle caratteristiche più sorprendenti della produzione di Marguerite Duras. I casi più eclatanti, quelli in cui uno stesso materiale narrativo, tematico o testuale viene ripreso in un genere o un sottogenere diverso da quello della prima apparizione, sono stati messi in evidenza. Sono stati invece trascurati i casi più sottili, quelli in cui la scrittrice è intervenuta puntualmente su uno dei suoi libri in occasione di una ristampa o di un passaggio in edizione tascabile. Certo è che la maggior parte di queste modifiche erano passate completamente inosservate prima della recente pubblicazione delle Opere complete dell'autrice. Queste riscritture minori, sebbene siano molto spesso "semplicemente" stilistiche, meritano di essere messe in relazione con i casi di riscrittura più emblematici dell'opera. Far emergere la loro natura, la loro portata, la loro influenza e le loro implicazioni, permette dunque di procedere ad alcuni adattamenti interpretativi, il cui interesse non si limita esclusivamente ai casi in questione. 